\title{
FORMULAE FOR WELL FORMED FORMULAE AND THEIR ENUMERATION
}

\author{
Dedicated to the memory of Hanna Neumann
}

DOV TAMARI

(Received 10 July 1972)

Communicated by M. F. Newman

\section{Introduction}

1. General Idea. In this paper formal operators and bracketings devices are essentially the same; so are well formed formulae and correct bracketings.

A well known theorem of symbolic logic (Post languages, etc.) and universal algebra characterizes well formed formulae among (linear) strings of symbols for variables and operators in terms of a system of numerical inequalities, one of them an equality. ${ }^{1}$

A better idea is to consider circular formulae. By bending formulae into oriented circles, or cycles, in particular well formed formulae into so-called unit cycles, one overcomes the asymmetry between head (left) and tail (right) end. Unit cycles have a simpler characterization than well formed formulae and are readily enumerated. The "welding point" can be recovered, as each unit cycle determines a unique cutting place such that the stretched out string is a well formed formula.

2. Resume. The basic results of this paper are

THEOREM 1. Each string of symbols satisfying $L-a=1$, or, what is the same, $a=m+n$ has one and only one cyclic permutation for which it is a well formed formula; that is $a=m+n$ is a necessary and sufficient condition for a cyclic formula to be that of a well determined well formed formula.

Here $L=n+m+1$ is the length of the string, $n$ the number of variables $x_{1}, \cdots, x_{n} ; m+1$ the number of operators $g_{0}, \cdots g_{m}$ of "arities" $a_{0}, \cdots, a_{m}$ respec-

1 The so-called Polish or Lukasiewicz notation, often somewhat misleadingly said to be "bracketfree" or "to rid us of the nuisance of brackets", provides a redundancy-free method of bracketing. 
tively; $a=\sum_{j=0}^{m} a$, the total arity of the string. The theorem assures that each unit cycle admits a unique cut determining a well formed formula. This gives a simple method of producing well formed formulae, indeed all well formed formulae. The essential tools for assuring the unity of the cut are Lemma 2, which asserts the fundamental bracketing property, and its corollary-the natural partial order of brackets by inclusion. As a further corollary one obtains

THEOREM 2. The number of distinct well formed formulae

$$
N_{g 0}^{(n)} \cdots g_{m}=\prod_{j=1}^{m}(n+j) / \prod_{f \in F} m_{f} \text { ! }
$$

where $F$ is the set of operations and $m_{f}$ the number of times the operator $f$ appears among the operators $g_{j}$.

Theorem 1 has two distinct proofs: The first one, based on building up well formed formulae from shorter ones has already been described above. The other one is essentially a proposition about sequences of integers and their partial sums (Proposition 2) which, by the way, has obvious generalizations to finite sums of reals (Proposition 3) and integrals (Proposition 4); this method effectively determines the unique place of the cut, that is, the cyclic permutation in Theorem 1, by using the existence of partial sums having an extremal property.

3. Interpretation and Extensions. Traditionally $F$ in $(*)$ would be interpreted as the set of "fundamental" operations (the $f$ 's not listed among the $g_{j}$ having multiplicity $m_{f}=0$ ); or, as the set of fundamental operations appearing among the $g_{j}$ (all $m_{f} \geqq 1$ ). However, the limitation to fundamental operations is irrelevanteverything applies to all operations, fundamental or derived (composed) ones, without distinction. Moreover, the interpretation as operations, i.e. as mappings $f: A^{a} \rightarrow A$ of the $a$-th power of a carrier (support) $A$ into $A$ ( $a=a_{f}$ is the arity of $f$ ), is irrelevant too. Our theory is one of abstract groupings, packings, or, synonymously, of bracketings as purely formal operations.

Extension of these methods to languages with richer logical content, admitting a greater variety of symbols (predicates, quantifiers, allowing for the special role of "=", etc.) is desirable and seems feasible. Further combinatorial and number theoretical results and applications to systems of linear inequalities, convex polyhedra etc., may be implied. One may also venture the suggestion of an analogue in theoretical physics: particle formation, by packing of sub-particles, and splitting of particles, considering well formed formulae built up from shorter ones as analogues of stable particles composed from smaller ones.

4. Background.-Formula (*) and many of its numerous interpretations, e.g. enumerating trees, in particular some of its special cases (binary bracketings or trees, or, what is the same, non-associative "powers") are not new. What 
seems to be new is the surprisingly simple Theorem 1, its elegant conceptual proof, as well as the more general interpretation.

This paper was motivated by the search for a simpler argument to replace the ingenuous, but long and difficult proof of a formula for the number of faces of given form of polyhedra $\mathscr{P}_{n}$ due to Mme de Fougères ([3], section 3, Theorem 2).

The idea of circular permutations was conceived independently and earlier, perhaps for the first time, by Silberger $[7]^{2}$ in connection with the special problem of enumeration of binary bracketings. The method of partial sums with an extremal property implying also cyclic permutations, appears, probably for the first time in Raney's [5 $]^{3}$ proof of formula (*). The history of special cases and interpretations of this problem goes back for more than 200 years, at least till Euler. Closed enumeration formulae were first proven, mostly by function-theoretical methods, in the 19th century (Binet, Catalan, Lamé, Rodriguez). Cayley and Schröder considered certain variations on this problem. Formula (*) was obtained for the first time by Erdelyi and Etherington in 1940 [2] (by function-theoretical means). Further references can be found in the books and papers listed in our bibliography and in standard reference works.

\section{Cyclic formulae}

1. Notations. One denotes: $x, x_{1}, \cdots, x_{n}$ variables (constants which are the same as nullary operations, included) which are symbols of "arity" 0 (or valency or weight 1), chosen from a set $X$;

$\{\cdots, f, \cdots\}=F=\bigcup_{a \in N}, F_{a}$ the set of functions or operations, $F_{a}$ the set of those of arity $a\left(N^{\prime}=\{1,2, \cdots\}\right)^{4} ; g_{0}, \cdots, g_{m}$ are $m+1$ not necessarily distinct $f \in F$;

$Y=X \cup F$ set of symbols $y$, from which $y_{1}, \cdots, y_{L}$ are chosen; $a_{y}$ arity of $y ; s=s^{L}=s_{1}^{L}=y_{1} \cdots y_{j} \cdots y_{L} \in Y^{L}$ a (linear) string of length $L, y_{1} / y_{L}$ its head/ end (first/last symbol) $S=\bigcup_{L \in N} Y^{L}$ set of strings ${ }^{5} ; L=n+m+1 ; a_{j}=a_{g_{j}}$, $\sum_{j=1}^{L} a_{y_{j}}=\sum_{j=0}^{m} a_{j}=a$ the total arity of $s$;

$v: S \rightarrow Z$ (integers) is the valency of strings defined by $v(y)=1-a_{y}, v(s)$ $=\sum_{j=1}^{L} v\left(y_{j}\right)=L-a$.

Operators act on symbols to their right; e.g. the distributive law for rings $x \cdot(y+z)=(x \cdot y)+(x \cdot z)$ will be written $\cdot x+y z=+\cdot x y \cdot x z$.

2. Some Preliminaries on Well Formed Formulae. A well known theorem of symbolic logic (Post Languages) and universal algebra (for example [1],

2 The author is obliged to Professor J. Isbell for calling his attention to this recent paper.

3 The author is obliged to Professor Don Knuth for this reference, as well as that to his recent book [4].

4 Putting $X=F_{\text {o }}$ one replaces $N^{\prime}$ by $N=[0,1,2, \cdots]$ and obtains $Y=F$.

5 Including the empty string $s_{0}$. 
p. 118; [6], pp. 152-157, p. 207) characterizes the subset $W, W \subset S$, of meaningful words (derived functions, polynomials) or well formed formulae (expressions substitutable for variables) by

$$
s \in W \Leftrightarrow v\left(s_{j}\right) \leqq 0 \quad(1 \leqq j<L), \quad v\left(s^{L}\right)=1 .
$$

This is a characterization by initial segments. Dually, their complements or final segments satisfy $v\left(C s^{j}\right)=v\left(y_{j+1} \cdots y_{L}\right) \geqq 1$ for all $j=0,1 \cdots, L-1$ with $C s^{0}=s=s^{L}$ and $v(s)=1$.

Defining a function $u=u_{s}: J^{L} \rightarrow Z\left(J^{L}=\langle 1, \cdots, L\rangle\right)$ assigning $j \leftrightarrow u_{s}(j)$ $=v\left(s_{j}\right)$ one reformulates this characterization as

Proposition 1. $s \in W \Leftrightarrow u_{s}(j)$ takes the maximum value 1 , and this only once at the "end" $j=L$.

Call a sequence $z_{1} \cdots, z_{L}$ of integers $\leqq 1$ with sum $=1$ a unit sequence, and call it well balanced if all properly partial, i.e. initial sums are $\leqq 0$. One reformulates

Proposition $1^{\prime} . s \in W \Leftrightarrow v\left(y_{1}\right), \cdots, v\left(y_{L}\right)$ is a well balanced unit sequence.

3. Cycles. Bend $s$ into an oriented cycle $t$ with adjition and subtraction of indices in $J^{L} \bmod L$. Substrings (segments or arcs) of length $l \leqq L$ are strings $s_{i}^{i-1+l}=y_{i}, \cdots, y_{i-1+l}$, the "full" arcs $s_{i}^{i-1}(V i \leqq L)$ having length $L$.

The common valence of $v\left(s_{i}^{i-1}\right)$ for all $i$ is the valenzy $v(t)$ of $t$. All cycles considered shall have positive valency $v(t)>0$. If $v(t)=1$ call $t$ a unit cycle.

If $s_{i}^{i-1+l} \in W$ call $s_{i}^{i-1+l}$ an elementary unit (e.u.).

LEMMa 1. Each $y_{i}$ of $t$ is head of a uniquely determined elementary unit $s_{i}^{i^{\prime}}$ (of length $l_{i}=i^{\prime}-i+1 \geqq 1$ ).

Proof. a) $y_{i} \in X \Rightarrow y_{i}=s_{i}^{i}$ is the desired elementary unit with $l_{i}=1$.

b) $y_{i} \in F \Leftrightarrow v\left(y_{i}\right)<1, v\left(s_{i}^{i-1}\right) \geqq 1 \Rightarrow \exists l(1<l \leqq L) \mid v\left(s_{i}^{i-1+l}\right)=1$;

$$
l_{i}=\min \left\{l \mid v\left(s_{i}^{i-1+l}\right)=1\right\}>1 .
$$

COROLlaRY 1. $t$ is covered by elementary units.

COROLlary 2. $i \leftrightarrow i^{\prime}$ defines a well defined function $J^{L} \rightarrow J^{L}$.

LEMMA 2. (Fundamental bracketing property): Elementary units do not overlap (properly); that is two distinct elementary units are either disjoint or one is contained in the other.

Proof. Assume e.u. $s_{i}^{i^{\prime}}, s_{j}^{j^{\prime}} i<j$, overlap properly, that is $i<j \leqq i^{\prime}<j^{\prime}$. This implies $v\left(s_{i}^{j-1}\right), v\left(s_{j}^{i^{\prime}}\right) \leqq 0$ and, therefore, the contradiction $1=v\left(s_{i}^{i^{\prime}}\right)=$ $v\left(s_{i}^{j-1}\right)+v\left(s_{j}^{l^{\prime}}\right) \leqq 0$. 
The following reformulation of this proof is even simpler and more conceptual: An intersection of two properly overlapping elementary units is simultaneously a final and an initial segment, that is of weight $\geqq 1$ and $\leqq 0$ which is absurd.

REMARK. There can be two intersections at once.

Corollary 3. Among the $L$ distinct full arcs $s_{i}^{i-1}$ there is at most one elementary unit.

Proof. Any two among them overlap (even twice).

A necessary condition for $t$ to have a full elementary unit is $v(t)=1$; by Corollary 3 there can be at most one. One proves that indeed there always is one. Consider the system of all elementary unit arcs of $t$ ordered by inclusion.

COROllary 4. $t$ is partitioned by its maximal elementary units.

COROLlaRY 5. $v(t)=L-a=$ number of maximal elementary units of $t$.

Corollary 6. $v(t)=L-a=1 \Leftrightarrow \exists i \mid s_{i}^{i-1} \in W$.

This proves

THEOREM 1. $v(t)=1 \Leftrightarrow t$ has a full elementary unit (of course, only one). As $L=n+m+1$ and $L-a=1 \Rightarrow a=n+m, v(t)=1 \Leftrightarrow a=n+m$. This gives the version of this theorem in the introduction. Other versions are:

THEOREM $1^{A}$. Each unit cycle admits one and only one cut that is, determines $a$ unique head at $i$ such that $s=s_{i}^{i-1}$ is a well formed formula. In other words:

For each string $s=y_{1} \cdots y_{L}$ with $v(s)=1$ there exists one and only one cyclic permutation such that $s_{i}^{i-1}=y_{i} \cdots y_{L} y_{1} \cdots y_{i-1} \in W$.

How to find $i$ ? If the cut falls between $y_{j-1} y_{j}, y_{L} y_{1}$ included, then $y_{j-1} \in X$ and $y_{j} \in F$. If there is only one such place this one is it. Otherwise one can try such places one after the other. Proposition 2 in the next section gives an independent characterization and direct determination of $i$.

4. Arithmetical Version and Generalizations. An elementary arithmetical version of theorem 1 is

THEOREM $1^{B}$. Every unit sequence admits one and only one cyclic permutation making it well balanced.

Proposition 1 and version $B$ of Theorem 1 suggest the stronger Proposition 2 with an even simpler proof. 
Proposirion 2. Let $z=z_{1}, \cdots, z_{L}$ be a unit sequence, $i-1$ the lowest index at which its (initial) partial sums take their maximal value: then $z_{i}, \cdots, z_{L}, z_{1}$, $\cdots, z_{i-1}$ is the unique well balanced sequence among the cyclic permutations of $z$.

Proof. Put $M=\sum_{j=1}^{i-1} z_{j}$. Then $\sum_{j=1}^{k} z_{j}<M$ for all $k<i-1, \sum_{j=1}^{k} z_{j} \leqq M$ for all $k$.

Therefore $\sum_{j=i}^{k} z_{j}=\sum_{j=1}^{k} z_{j}-M \leqq 0 \quad i \leqq k \leqq L$

$$
\sum_{j=i}^{L} z_{j}+\sum_{j=1}^{k} z_{j}=1-M+\sum_{j=1}^{k} z_{j} \begin{cases}\leqq 0, & k<i-1 \\ =1, & k=i-1 .\end{cases}
$$

One obtains the uniqueness, as above from the fundamental bracket property (Lemma 2), that is, by Corollary 3; or independently, as follows:

For $l \neq i$ the sequence $z_{l}, \cdots, z_{L}, z_{1}, \cdots, z_{l-1}$ will not be well balanced because

for $l<i: \sum_{j=l}^{i-1} z_{j}=\sum_{j=1}^{i-1} z_{j}-\sum_{j=1}^{l-1} z_{j}>0$,

for $l>i: \sum_{j=1}^{L} z_{j}+\sum_{j=1}^{i-1} z_{j}=1-\sum_{j=1}^{l-1} z_{j}+\sum_{j=1}^{i-1} z_{j} \geqq 1$.

An obvious, but weak generalization is

Proposition 3. Let $r_{1}, \cdots, r_{L}$ be a sequence of real numbers with positive sum $s>0$ and $i-1$ the first index at which the partial sums take their greatest value. Then all properly partial sums of the cyclic permutation

$$
r_{i}, \cdots, r_{L}, r_{1}, \cdots, r_{1-1}
$$

are $<s$; that is, its sequence of partial sums takes the smallest possible greatest value namely s, and this only once namely as the total sum.

REMARKs. The first $L-i+1$ partial sums are even $\leqq 0$. The $i$ of the proposition is well defined, but there is no point in emphasizing uniqueness as no generalization of well balanced has been formulated. If all $r_{i}$ are integral multiples of $s$ and $\leqq s$; one falls back to Proposition 2 . Otherwise new, possibly richer possibilities arise.

An even wider generalization is

Proposition 4. Let $v$ be a real function in the interval $a \leqq x \leqq a+L$ with well defined integral

$$
V(x)=\int_{a}^{x} v \text { for all } x a \leqq x \leqq a+L
$$

and $s=V(a+L)>0 ;$ let $b, a<b \leqq a+L$, be the first $(=$ smallest $) x$ at which $V(x)$ reaches its greatest value. Put 


$$
u(x)=\left\{\begin{array}{l}
v(x) \quad \text { for } b \leqq x \leqq a+L \\
v(x-L) \text { for } a+L<x \leqq b+L
\end{array}\right.
$$

and

$$
\begin{gathered}
U(x)=\int_{b}^{x} u \quad b \leqq x \leqq b+L . \\
\text { Then } U(x)=\left\{\begin{array}{lll}
<s & \text { for } & x<b+L \text { (even) } \\
\leqq 0 & \text { for } & x \leqq a+L \\
=s & \text { for } & x=b+L .
\end{array}\right.
\end{gathered}
$$

Proof. Obvious.

\section{Enumeration of well for ned formulae}

1. First derivation. - Consider $x_{1}, \cdots, x_{n}$ as $n$ distinct points in cyclic order determining $n$ places, place $p$ being the open arc from $x_{p-1}$ to $x_{p}$ (for $p=1$ from $x_{n}$ to $\left.x_{1}\right)$. The operators $g_{0}, \cdots, g_{m}$ are points in these places: $g_{0}$ is in one of $n$ possible places, dividing in into two new places; $g_{1}$ in one of the $n+1$ places determined by $x_{1}, \cdots, x_{n}$ and $g_{0}$, dividing again one of them into two; etc.; finally $g_{m}$ can be placed in any of $n+m$ places determined by $x_{1}, \cdots, x_{n}$ and $g_{0}, \cdots, g_{m-1}$ One has therefore

$$
n(n+1) \cdots(n+m)=\prod_{j=0}^{m}(n+j)
$$

possible arrangements of cyclic formulae with $m+1$ operators with respect to the fixed cycle $\left(x_{1}, \cdots, x_{n}\right)$.

Cycles, distinct only by permutations of places of repeated appearances of the same operator, are identical. If $f \in F$ appears $m_{f}$ times among the $g\left(\sum_{f \in F} m_{f}\right.$ $\left.=m+1, \sum_{f \in F} a_{f} m_{f}=a\right)$, the number of distinct cycles is

$$
\prod_{j=0}^{m}(n+j) / \prod_{f \in F} m_{f} !
$$

If $a=n+m$, the cycles are unit cycles, each determining a unique well formed formula with a certain $x_{i}(i=1, \cdots, n)$ as its first variable $\in X$. Therefore $N_{g_{0}}^{(n)} \cdots g_{m}$, the number of well formed formulae with indeterminates $x_{1}, \cdots, x_{n}$ in this order, i.e. with $i=1$, is $1 / n$ of (2):

THEOREM 2.

$$
N_{g_{0} \cdots g_{m}}^{(n)}=\prod_{j=1}^{m}(n+j) / \prod_{f \in F} m_{f} !
$$


2. Alternative derivation of formula (*). The number of strings in $L=n+m+1$ distinct symbols $x_{1}, \cdots, x_{n}, g_{0}, \cdots, g_{m}=(n+m+1)$ !

The $n$ variables are interchangeable as their notation or enumeration is indifierent; so are the $m_{f}$ operators $g_{j}$ equal $f$. Therefore there are only $(n+m+1)$ ! $/ n ! \Pi m_{f}$ ! distinct strings. One and only one among the $n+m \cdot+1$ cyclic permutations of each string is a well formed formula. Therefore one has precisely

$$
N_{g 0 \cdots g}^{(n)}=(n+m) ! / n ! \Pi m_{f} !=\prod_{j=1}^{m}(n+j) / \prod_{f \in F} m_{f} !
$$

distinct well formed formulae as above.

3. Applications of (*). Formula (3) is nearly identical with that of Theorem 2 of [3];

$$
f_{d_{0} \cdots d_{i}}^{(n)}=\prod_{j=1}^{m}(n+2+j) / \prod_{j=0}^{\infty} t_{j} !
$$

where $t_{j}$ is the number of $d_{k}=j$, that is, $\sum_{j=0}^{\infty} t_{j}=i+1$ and $\sum_{j=1}^{\infty} t_{j}=n-i$. (**) is (*) with $F=\{f \mid j=2,3, \cdots\}, f$ just the "universal" $j$-ary operation (ignoring the unary identity map $f_{1}$ ). In (**) $n$ is replaced by $n+2, m$ by $i, m_{j+2}$ by $t_{j}$, and $g_{k}$ by $d_{k}$ with $a_{k}=d_{k}+2$.

REMARK. One interprets the universal $j$-ary operation $f_{j}$ as " $j$-ary brackets" (this, indeed, is meant in [3]), i.e. the formation of $j$-uples. One extends the carrier $A$ to $A \cup A \times A \cup \cdots=\bigcup_{j=1}^{\infty} A^{j}=\mathfrak{A} ; f_{1}$ is extended from $A$ to $\mathfrak{H}$, and so are all $f_{j}$.

One obtains the number of binary bracketings $k_{n}$ by putting $F=\{f\}, f=f_{2}$ the universal binary operation,

$$
\sum m_{f}=m_{f}=m+1, a=2 m_{f}=n+m \rightarrow n+m=2 n-2,
$$

that is

$$
k_{n}=\underbrace{N_{m=2}^{(n)}}_{m+1=n-1 \text { times }}=(n+m) ! / n ! \Pi m_{f} !=\frac{(2 n-2) !}{(n-1) ! n !}=\left(\begin{array}{c}
2(n-1) \\
n-1
\end{array}\right) / n .
$$

\section{Bibliography}

[0] A. Cayley: 'On the analytical forms called trees', 2nd part, Coll. Math. Papers, vol. 4, Cambride 1891, 114-5.

[1] P. M. Cohn: Universal Algebra, (Harper and Row, 1965).

[2] A. Erdélyi and I. H. M. Etherington: 'Some problems of non-associative combinations (2)', Edinburgh Math. Notes, 32 (1940), VII-XII.

[3] D. de Fougères and D. Tamari: 'La structure polyèdrale des complexes de parenthésages', (submitted). 
[4] Don Knuth: Fundamental Algorithms, (Addison-Wesley, 1969.)

[5] G. N. Raney: 'Functional composition patterns and power series reversion', Trans. Amer. Math. Soc. 94 (1960), 441-51.

[6] P. C. Rosenbloom: The elements of mathematical logic. (Dover 1950.)

[7] D. M. Silberger: 'Occurrence of the integer (2n-1)!/n! (n-1)!'; Prace Mat. 13 (1969), 91-96; M. R. 40 \# 2556.

[8] D. Tamari: Monoides préordonnés et chaînes de Malcev; Thèse, (Paris 1951) (printed part in Bull. Soc. Math. France 82 (1954), 53-96).

[9] D. Tamari 'The algebra of bracketings and their enumeration', Nieuw Arch. Wisk. X (1962), 131-146.

Department of Mathematics

State University of New York at Buffalo

4246 Ridge Lea Road, Amherst, N.Y. 14226

U.S.A. 